\title{
Stray Cats Gastrointestinal Parasites and its Association With Public Health in Ahvaz City, South Western of Iran
}

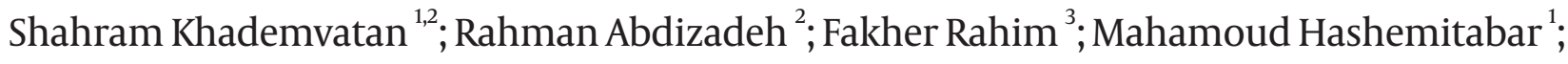 \\ Mohammad Ghasemi ${ }^{2}$; Mahdi Tavalla ${ }^{2,}$ \\ ${ }^{1}$ Cellular and Molecular Research Center, Ahvaz Jundishapur University of Medical Sciences, Ahvaz, IR Iran \\ ${ }^{2}$ Department of Medical Parasitology, Ahvaz Jundishapur University of Medical Sciences, Ahvaz, IR Iran \\ ${ }^{3}$ Toxicology Research Center, Ahvaz Jundishapur University of Medical Sciences, Ahvaz, IR Iran \\ ${ }^{*}$ Corresponding author: Mahdi Tavalla, Department of Medical Parasitology, Jundishapur University of Medical Sciences, P.O. Box: 613715794, Ahvaz, IR Iran. Tel: +98-6113367543-50, \\ Fax:+98-6113332036, E-mail: am.tavalla@gmail.com
}

Received: March 5, 2013; Revised: July 16, 2013; Accepted: September 21, 2013

\begin{abstract}
Background: Cats are the hosts for some zoonotic parasites such as Toxoplasma gondii and Toxocara spp. which are important in medicine and veterinary. Studies on the prevalence of intestinal parasites of cats have received little attention in south west of Iran.

Objectives: The current study aimed to investigate the prevalence of parasites in stray cats in Ahvaz.

Materials and Methods: Random sampling was carried out from January to May 2012. One hundred and forty fecal samples from stray cats were examined using sucrose flotation method.

Results: Gastrointestinal parasites were found in 121 of the $140(86.4 \%)$ examined samples. The parasites detected in stray cats were Toxocara spp. (45\%, 63/140), Isospora spp. (21.4\%, 30/140), nematode larvae (21.4\%, 30/140), Taenia spp. (18.6\%, 26/140), Sarcocystis spp. (17.1\%, 24/140), Eimeria spp. (15\%, 21/140), Blastocystis spp.(14.3\%, 20/140), Giardia spp, (10.7\%,15/140), Physaloptera spp. (7.1\%, 10/140), and amoeba cyst $(5.7 \%, 8 / 140)$ respectively. The prevalence of infection by Joyexiella spp. and hook worms (4.3\%, 6/140), for example, Dipylidium caninum (2.9\%, 4/140) was similar; and the prevalence of infection by T. gondii and Dicrocoelium dendriticum was similar (1.4\%, 2/140).

Conclusions: Since the prevalence of zoonotic gastrointestinal parasites such as Toxocara spp. in stray cats is high, there is a need to plan adequate programs to control these zoonotic parasites.
\end{abstract}

Keywords: Gastrointestinal Parasites; Stray Cat; Zoonoses; Iran

\section{Background}

In recent years, the importance of potentially zoonotic organisms posed by stray cats and its importance in public health has been considered (1). Cats and other felines, can act as reservoirs, carriers, transmitters and definitive hosts for many intestinal parasites. These animals play an important role in parasite transmission to human and other animals by shedding parasitic eggs, larvae, cyst or oocyst in their feces $(2,3)$. Furthermore, cats have significant role in contamination of environment with discharge helminths eggs and protozoan cysts into the public environments $(4,5)$. Occupational groups such as gardeners, farmers, construction and municipality workers, also toddlers and small children who play in parks or in contaminated soil are classified as high-risk groups for many zoonotic parasitic infections (6-8). Toxoplasma gondii and Toxocara cati are the most important zoonotic feline gastrointestinal parasites which can be transmitted to human without the involvement of vectors or intermediate hosts. Therefore, humans are directly infected (can be directly infected) by contact with sporulated oocyst of T. gondii and embryonated egg of Toxocara spp. $(7,9,10)$.

The most common way for human toxoplasmosis is through accidentally ingesting oocyst-contaminated soil shed by infected cats or by ingesting tissue cysts present in undercooked meat (4). According to previous reports, zoonotic nematodes such as T. cati and Ancylostoma tubaeform are the most common intestinal helminths parasites of cats worldwide (4). Furthermore other parasitic infections including Giardia duodenalis, Cryptosporidium spp., Sarcocystis spp., Blastocystis spp., Microsporidia spp., Echinococcus multilocularis, Ancylostoma spp. and Strongyloides spp. have zoonotic potential importance in felines $(3,5,11-16)$.

In Iran, cats often live freely in urban areas and in human houses as a predator of rats. The growing number of stray cats in urban residential areas and their easy access to public environment in order to get their nutritional needs from garbage may contaminate soil, food and

Implication for health policy/practice/research/medical education:

Cats as the hosts for some zoonotic parasites such as Toxoplasma and Toxocara are important in medicine and veterinary. Since studies on the prevalence of intestinal parasites of cats have received little attention in south west of Iran, the current study aimed to investigate the prevalence of parasites in stray cats in Ahvaz, Khuzestan Province, IR Iran.

Copyright (c) 2014, Ahvaz Jundishapur University of Medical Sciences; Published by Kowsar Corp. This is an open-access article distributed under the terms of the Creative Commons Attribution License, which permits unrestricted use, distribution, and reproduction in any medium, provided the original work is properly cited. 
water, and consequently increase parasitic infections, in humans. Due to the close association of cats with human in urban areas, and since comprehensive studies on zoonotic parasites of cats have received little attention in Iran, the current study was designed to predict the prevalence of gastrointestinal parasites of stray cats in Ahvaz, city south western of Iran.

\section{Objectives}

The current study aimed to investigate the prevalence of parasites in stray cats in Ahvaz, Khuzestan Province, Iran.

\section{Materials and Methods}

\subsection{Study Area}

Ahvaz, capital of Khuzestan province in southwest Iran, (Figure 1) $\left(31^{\circ} 19^{\prime} \mathrm{N}, 41^{\circ} 09^{\prime} \mathrm{E}\right)$ is characterized by a hot and humid climate. This city is known to have the hot test temperatures on record for a populated city anywhere in the world. This city is more than $200 \mathrm{~km}^{2}$, and approximate population of 1200000 (17).

\subsection{Fecal Samples Collection}

At first, the town was geographically divided into five regions: north, south, east, west and center. During January to May 2012 one hundred and forty discharged cat fecal samples were collected by simple random selection from different parts of the public places in Ahvaz including parks, squares and children playgrounds. Samples were put in polyethylene bags marked according to geographical area, date of collection and consistency. The samples of stray cats feces were separately carried to the laboratory and kept at $4^{\circ} \mathrm{C}$ until processing.

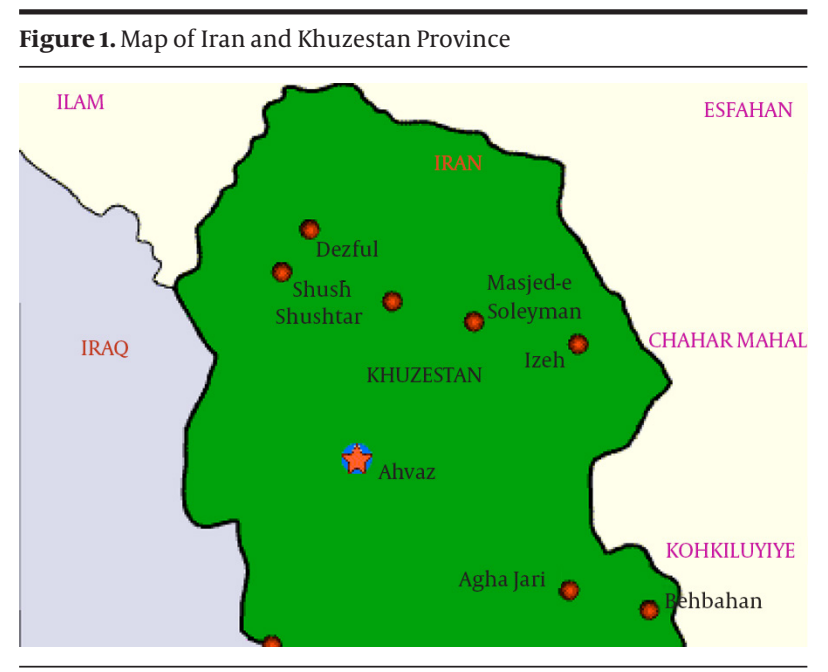

Ahvaz distinguished by star sign, based on the map of Khuzestan province.

\subsection{Parasitological Procedure}

Eggs, oocysts and other parasitic forms were isolated for each cat feces by saturated sucrose solution described previously by Sheather with some modification (18). Briefly, 3-5 g of any fecal samples was stirred into $50 \mathrm{~mL}$ normal saline until completely dispersed. The suspension was passed through four unsterile gauze layers to separate large particles. The suspension was transferred into $50 \mathrm{~mL}$ falcon tube and centrifuged at $2000 \mathrm{rpm}$ for 5 minutes. Then supernatant was removed and the sediment was washed with distilled water twice. Sediment was gently added to new falcon tube that contained 15 mL saturated sugar (Merck, Germany) solution with specific gravity of $1.30 \mathrm{~g} / \mathrm{mL}$ and centrifuged at $800 \mathrm{~g}$ for 5 minutes. The interface and the upper layer of liquid were transferred to a new tube and this falcon was filled with distilled water and centrifuged at $1000 \mathrm{rpm}$ for $5 \mathrm{~min}$ utes. Sediment was washed until removal of sucrose residues. Finally the sediment was evaluated for parasites to identify their morphological characteristics via light microscopy at $\times 100$ and $\times 400$ magnifications accordingly (19).

\subsection{Statistical Analysis}

Statistical analysis was performed by SPSS 16.0 software. The prevalence and confidence intervals (C.I) of each parasite were calculated. Difference between groups was determined by Chi-square test, with the significance level of $\mathrm{P}=0.05$.

\section{Results}

Of the 140 samples of feline feces collected from stray cats, 121 samples (86.4\%) were infected with at least one gastrointestinal parasite. The results of the study showed that the prevalence of helminth infection was higher than protozoan parasite. In the current study fifteen genera of intestinal parasites were detected in stray cats stool samples. Types of helminthic infections included three genera of nematodes, Toxocara spp. (45\%; 95\% CI: 35 55\%), Physaloptera spp. (10\%; 95\% CI:3-14\%) and hookworms (4.3\%; 95\% CI:1.4-10\%), three genera of cestodes, Taenia spp. (18.6\%; 95\% CI:11-27\%), Joyexiella spp. (4.3\%: 95\% CI: 1.4-10\%), Dipylidium spp. (2.9\%; 95\% CI: 0.7-9\%) and one species of Trematode, Dicrocoelium dendriticum (1.4; 95\% CI: 0.1-6). Furthermore, seven genera of protozoa were detected in the collected samples from different geographical areas of Ahvaz including Isopora spp. (21.4\%; 95\% CI: 14-30\%), Giardia spp. (10.7\%; 95\% CI: 5-18\%), Sarcocystis spp. (17.1\%; 95\% CI: 10-26\%), T. gondii (1.4\%; 95\% CI: 0.1-6\%), Amoeba cyst (5.7\%; 95\% CI: 2.2-12\%), Eimeria spp. (15\%; 95\% CI: 8.9-23\%) Blastocystis (14.3\%; 95\% CI: 8 23\%) and other oocysts (7.9\%; 95\% CI: 3.7-15\%) (Table 1). In the current study, 86 samples (61.4\%; 95\% CI: 51-70\%) were infected with multiple gastrointestinal parasites. Results of parasites co-infection 
Khademvatan S et al.

Table 1. Distribution of Gastrointestinal Parasites in the Feces of Stray Cats Collected From Public Environments in Different Geographical Areas of Ahvaz ${ }^{\text {a }}$

\begin{tabular}{lcccccc}
\hline Parasite species & North & South & East & West & Center & Total \\
\hline Toxocara spp. & $14(22.2)$ & $9(14.3)$ & $13(20.6)$ & $9(14.3)$ & $18(28.6)$ & $63(100)$ \\
Isopora spp. & $4(20)$ & $7(23.3)$ & $7(23.3)$ & $5(16.7)$ & $5(16.7)$ & $30(100)$ \\
Giardia spp. & $5(33.3)$ & $3(20)$ & $4(26.7)$ & 0 & $3(20)$ & $15(100)$ \\
Sarcocystis spp. & $6(25)$ & $6(25)$ & $3(12.5)$ & $4(17.7)$ & $5(20.8)$ & $24(100)$ \\
Toxoplasma gondii & $1(50)$ & 0 & 0 & 0 & $1(50)$ & $2(100)$ \\
Joyexiella spp. & $1(16.7)$ & $16.7(1)$ & $33.3(2)$ & $2(33.3)$ & 0 & $6(100)$ \\
Dicrocoelium dendriticum & $1(50)$ & $50(1)$ & 0 & 0 & 0 & $2(100)$ \\
Physaloptera spp. & $1(10)$ & 0 & $3(30)$ & $3(30)$ & $3(30)$ & $10(100)$ \\
Amoeba cyst & $5(62.5)$ & $1(12.5)$ & $1(12.5)$ & 0 & $1(12.5)$ & $8(100)$ \\
Taenia spp. & $4(15.4)$ & $26.9(7)$ & $4(15.4)$ & $5(19.2)$ & $6(23.1)$ & $26(100)$ \\
Dipylidium caninum & 0 & 0 & $2(50)$ & $1(25)$ & $1(25)$ & $4(100)$ \\
Nematode larvae & $3(10)$ & $4(13.3)$ & $7(23.3)$ & $8(26.7)$ & $8(26.7)$ & $30(100)$ \\
Eimeria spp. & $6(28.6)$ & $3(14.3)$ & $5(23.8)$ & $3(14.3)$ & $4(19)$ & $21(100)$ \\
Blastocystis & $5(25)$ & $4(20)$ & $3(15)$ & $5(25)$ & $3(15)$ & $20(100)$ \\
Hook worms & $2(33.3)$ & $11(6.7)$ & $1(16.7)$ & $1(16.7)$ & $1(16.7)$ & $6(100)$ \\
\hline Daran
\end{tabular}

${ }^{\mathrm{a}}$ Data are presented as No.(\%).

\begin{tabular}{lcc}
\hline \multicolumn{2}{l}{ Table 2. Distribution of Positive Parasitic Co-Infection in Stool of Stray Cats Collected From Ahvaz ${ }^{\text {a }}$} & \\
\hline Number of Parasite in Each Sample & Results & CI (95\%) \\
\hline $\mathbf{1}$ & $35(25)$ & $17-34 \%$ \\
$\mathbf{2}$ & $43(30.7)$ & $22-40 \%$ \\
$\mathbf{3}$ & $21(15)$ & $8.9-23 \%$ \\
$\mathbf{4}$ & $18(12.9)$ & $7.3-21 \%$ \\
$\mathbf{5}$ & $2(1.4)$ & $0.1-6.8 \%$ \\
$\mathbf{6}$ & $2(1.4)$ & $0.1-6.8 \%$ \\
\hline
\end{tabular}

a Data are presented as No.(\%).

Table 3. Number of Gastrointestinal Parasites in the Feces of Stray Cats Collected From Public Environments of Ahvaz, October 2011 to September $2012^{\mathrm{a}}$

\begin{tabular}{lcccc}
\hline \multirow{2}{*}{ Parasite Species } & \multicolumn{3}{c}{ Public Environment } & \multirow{2}{*}{ Total } \\
\cline { 2 - 4 } Toxocara spp. & Park & Square & Children's Playground & 63 \\
Isopora spp. & $11(17.5)$ & $7(11.1)$ & $45(71.4)$ & 3 \\
Giardia spp. & $7(23.3)$ & $3(10)$ & $9(66.7)$ & 15 \\
Sarcocystis spp. & $2(13.3)$ & $4(26.7)$ & $12(50)$ & 24 \\
Toxoplasma gondii & $6(25)$ & $6(25)$ & $1(50)$ & 2 \\
Joyexiella spp. & $1(50)$ & 0 & $3(50)$ & 6 \\
Dicrocoelium dendriticum & $1(16.7)$ & $2(33.3)$ & $1(50)$ & 2 \\
Physaloptera spp. & $1(50)$ & 0 & $6(60)$ & 10 \\
Amoeba spp. Cyst & $3(30)$ & $1(10)$ & $7(87.5)$ & 8 \\
Other oocyst & 0 & $1(12.5)$ & $6(54.5)$ & 7.9 \\
Taenia spp. & $3(27.3)$ & $2(18.2)$ & $18(69.2)$ & 26 \\
Dipylidium spp. & $5(19.2)$ & $3(11.5)$ & $1(25)$ & 4 \\
Nematode larvae & $2(50)$ & $1(25)$ & $21(70)$ & 30 \\
\hline Eimeria spp. & $8(26.7)$ & $1(3.3)$ & $14(66.7)$ & 21 \\
\hline Blastocystis & $4(19)$ & $3(14.3)$ & $15(75)$ & 20 \\
\hline Hook worms & $2(10)$ & $3(15)$ & $5(83.3)$ & 6 \\
\hline
\end{tabular}

${ }^{\mathrm{a}}$ Data are presented as No.(\%). 
revealed that 43 samples (30.7\%) were infected with at least two parasites (Table 2). The prevalence of intestinal parasites in stool samples of stray cats in different geographical areas of Ahvaz parks, squares and children playgrounds were $83.3 \%$, 90.5\% and $86.5 \%$, respectively (Table 3). Frequency of parasites based on consistency of stool samples did not show statistical significance between consistency and existence of parasites $(P>0.05)$.

\section{Discussion}

The prevalence of gastrointestinal parasites in cats can vary and it may be due to geographical region (temperature and humidity), season, behaviors and habits of the local animal populations and the type of population of cat (stray, feral, shelter, household) (8). In the current study the prevalence of parasites in stray cats fecal samples from different areas of city, was (86.4\%) that indicated cats were infected with various parasites especially zoonotic parasites. The findings of the current study are in agreement with other studies in different parts of Iran and the world such as North of Iran and northern region of Nile delta on Egypt that indicated 90\% and 91\% of stray cats were infected with intestinal parasites, respectively $(8,20)$. Also other investigations on the prevalence of intestinal parasites in cats of Ebro Valley, Spain and Rio de Janeiro indicated that $90 \%$ of cats from both areas were infected with parasites $(21,22)$.

In another study Summerfelt et al. reported that 58.3\% of the cat feces collected from open spaces of public institutions in Buenos Aires of Argentina were infected with intestinal parasites and the most common parasite was T. cati (61.2\%) (23). In Iran, population growth, particularly in large cities, in the recent decades is likely the cause to change the lifestyle and rapid expansion of apartment life and the tendency to spend the leisure time in public environments such as recreational areas, parks, squares and children playgrounds. Most of the Iranians are Muslims and according to religious recommendations they avoid keeping animals in their houses and touching them.

The growing number of stray cats in urban residential areas, the easy access of these animals to the public environments in order to meet their nutritional needs, and their tendency to discharge helminths eggs, larvae, and protozoan cysts by defecating in these locations may contribute to soil, food and water contamination. As there is limited knowledge about the life cycle and transmission ways ofzoonotic parasites in stray cats to people, the prevalence can be increasing and has led to an increase in the risk of zoonotic parasitic infections, such as toxoplasmosis and larvae migrants in humans especially children who play in such places and contact directly or indirectly with contaminated soil.

In the present study, the most frequent species of parasites was Toxocara spp. (45\%) which was similar to the prevalence of $T$. cati infection in stray cats recorded in other regions of Iran. Zibaei et al. reported that $42.6 \%$ of stray cats in Shiraz were infected with T. cati (24). But $28.8 \%, 13.3 \%$ and $8 \%$ of stray cats in Mashhad, Kashan and Zanjan were infected had toxocariasis, respectively (2527). In another study conducted in the north of Iran (Sari) prevalence of T. cati in stray cats was $44 \%$ (28). It appears that suitable environmental conditions including optimal temperature and higher humidity in Ahvaz and Sari are the possible reasons for the higher prevalence compared to the results of other studies conducted in Northeast, central and Northwest of Iran (25-27). Additionally, some studies on the prevalence of Toxocara spp. egg in soil of parks in Iran show that 5.8\%-63.3\% of soils were contaminated with Toxocara spp. egg (29-32).

Blaszkowska et al. indicated that $73.5 \%$ of soils of children playgrounds in Lodz of Poland were contaminated with Toxocara spp. The eggs of Toxocara spp. are resistant to environmental conditions and can remain infectious for years in a favorable environment, therefore young children who play in such locations are the main population at risk of toxocariasis due to geophagia, onychophagia and poor individual hygiene (33). In addition, the examination of samples collected from different locations of Ahvaz revealed that the prevalence of Giardia spp. in feces of stray cats was $10.7 \%$ which is lower than that of the stray cats in west of Iran, Ilam province (18.91\%) (34) while the prevalence rate of Giardia spp. in the present research is higher than those of Egypt (2\%) (8) and Kashan (0.9\%) (25).

The assemblages A and B of Giardia are zoonotic genotypes that can infect cats; therefore giardiasis in stray cats is a potential hazard for human. Paoletti et al. in order to determine the prevalence and genetic characterization of Giardia spp. in cats of Italy, investigated one hundred and eighty one feces samples of cats with IFA and polymerase chain reaction (PCR) techniques and the findings of this survey showed that $4.4 \%$ of cats were infected and the infected animals, including three cats that harbored Giardia duodenalis assemblage A, were infected with the feline-specific G. duodenalis assemblage $\mathrm{F}$ (13). Among the parasites found in the current survey Blastocystis spp. (14.3\%) and Sarcocystis spp. (17.1\%) are considered as important zoonotic infections that were similar to results of intestinal protozoa in Kashan but more than the prevalence of Sarcocystis spp. in Germany (0.3\%) (35).

The feces of stray cats in general environment are important as the potential source of gastrointestinal parasites from medical and veterinary point of view and play a major role in transmitting theses parasites through fecal contamination of soil, food or water. The results of the present study indicated that the prevalence of gastrointestinal parasites especially zoonotic parasites in feces of stray cats in Ahvaz was high. The free entrance of stray cats and defecation in public places increases the 
risk of zoonotic parasitic diseases such as toxocariasis and giardiasis in humans. Therefore it can be a potential hazard for humans and this phenomenon is an alert for municipality and health service centers. It is suggested that control programs including education for people about zoonotic parasites, preventing free entrance of stray cats and dogs in public places and collection and hygienic disposal of stray cats and dogs feces by municipal workers every day should be developed by mass media, health service centers and veterinarians.

\section{Acknowledgements}

This study was supported by grants from Ahvaz Jundishapur University of Medical Sciences.

\section{Authors' Contributions}

Mehdi Tavalla and Rahman abdizadeh and Shahram Khademvatan wrote the paper and thanks from Mahmoud Hashemitabar for consultation.

\section{Funding/Support}

This study is conducted and financially supported by grants from Ahvaz Jundishapur University of Medical Sciences. The funders had no role in study design, data collection and analysis, decision to publish, or preparation of the manuscript.

\section{References}

1. Hill SL, Cheney JM, Taton-Allen GF, Reif JS, Bruns C, Lappin MR. Prevalence of enteric zoonotic organisms in cats. J Am Vet Med Assoc. 2000;216(5):687-92.

2. Alves JM, Magalhaes V, Matos MA. [Toxoplasmic retinochoroiditis in patients with AIDS and neurotoxoplasmosis]. Arq Bras Oftalmol. 2010;73(2):150-4.

3. Mircean V, Titilincu A, Vasile C. Prevalence of endoparasites in household cat (Felis catus) populations from Transylvania (Romania) and association with risk factors. Vet Parasitol. 2010;171(1-2):163-6.

4. Sowemimo OA. Prevalence and intensity of gastrointestinal parasites of domestic cats in Ode-Irele and Oyo communities, Southwest Nigeria. J Parasitol Vector Biol. 2012;4(1):7-13:e11079.

5. Jamshidi S, Tabrizi AS, Bahrami M, Momtaz H. Microsporidia in household dogs and cats in Iran; a zoonotic concern. Vet Parasitol. 2012;185(2-4):121-3.

6. Abu-Madi MA, Behnke JM, Prabhaker KS, Al-Ibrahim R, Lewis JW. Intestinal helminths of feral cat populations from urban and suburban districts of Qatar. Vet Parasitol. 2010;168(3-4):284-92.

7. Duarte A, Castro I, Pereira da Fonseca IM, Almeida V, Madeira de Carvalho LM, Meireles J, et al. Survey of infectious and parasitic diseases in stray cats at the Lisbon Metropolitan Area, Portugal.J Feline Med Surg. 2010;12(6):441-6.

8. Khalafalla RE. A survey study on gastrointestinal parasites of stray cats in northern region of Nile delta, Egypt. PLoS One. 2011;6(7).

9. Dabritz HA, Conrad PA. Cats and Toxoplasma: implications for public health. Zoonoses Public Health. 2010;57(1):34-52.

10. Kulasena VA, Rajapakse RP, Dubey JP, Dayawansa PN, Premawansa S. Seroprevalence of Toxoplasma gondii in cats from Colombo, Sri Lanka. J Parasitol. 2011;97(1):152.

11. Awadallah MAI. Endoparasites of Zoonotic Importance. 2010.

12. Deplazes P, van Knapen F, Schweiger A, Overgaauw PA. Role of pet dogs and cats in the transmission of helminthic zoonoses in Europe, with a focus on echinococcosis and toxocarosis. Vet Parasitol. 2011;182(1):41-53.

13. Paoletti B, Otranto D, Weigl S, Giangaspero A, Di Cesare A, Traversa D. Prevalence and genetic characterization of Giardia and Cryptosporidium in cats from Italy. Res Vet Sci. 2011;91(3):397-9.

14. Thompson RC, Smith A. Zoonotic enteric protozoa. Vet Parasitol. 2011;182(1):70-8.

15. Tzannes S, Batchelor DJ, Graham PA, Pinchbeck GL, Wastling J, German AJ. Prevalence of Cryptosporidium, Giardia and Isospora species infections in pet cats with clinical signs of gastrointestinal disease. J Feline Med Surg. 2008;10(1):1-8.

16. Vassalos CM, Papadopoulou C, Vakalis NC. Blastocystosis: an emerging or re-emerging potential zoonosis? Vet Ital. 2008;44(4):679-84.

17. Census of the Islamic Republic of Iran. 2014. Available from: http://www.amar.org.ir.

18. Sheather AL. The detection of intestinal protozoa and mange parasites by a floatation technique. J Comp Pathol Ther:1923;36:266-75.

19. Soulsby EJL, Helminths A. Protozoa of Domesticated Animals Baillière Tindall. East Sussex, UK. 1982.

20. Sharif M, Daryani A, Nasrolahei M, Ziapour SP. A survey of gastrointestinal helminthes in stray cats in northern Iran. Comp Clin Pathol.2009;19(3):257-61.

21. Labarthe N, Serrao ML, Ferreira AM, Almeida NK, Guerrero J. A survey of gastrointestinal helminths in cats of the metropolitan region of Rio de Janeiro, Brazil. Vet Parasitol. 2004;123(1-2):133-9.

22. Serra CM, Uchoa CM, Coimbra RA. [Parasitological study with faecal samples of stray and domiciliated cats (Felis catus domesticus) from the Metropolitan Area of Rio de Janeiro, Brazil]. Rev Soc Bras Med Trop. 2003;36(3):331-4.

23. Sommerfelt IE, Cardillo N, Lopez C, Ribicich M, Gallo C, Franco A Prevalence of Toxocara cati and other parasites in cats' faeces collected from the open spaces of public institutions: Buenos Aires, Argentina. Vet Parasitol. 2006;140(3-4):296-301.

24. Zibaei M, Sadjjadi SM, Sarkari B. Prevalence of Toxocara cati and other intestinal helminths in stray cats in Shiraz, Iran. Trop Biomed. 2007;24(2):39-43.

25. Mohsen A, Hossein H. Gastrointestinal parasites of stray cats in Kashan, Iran. Trop Biomed. 2009;26(1):16-22.

26. Borji H, Razmi G, Ahmadi A, Karami H, Yaghfoori S, Abedi V. A survey on endoparasites and ectoparasites of stray cats from Mashhad (Iran) and association with risk factors. J Parasit Dis. 2011;35(2):202-6.

27. Esmaeilzadeh M, Shamsfard M, Kazemi A, Khalafi SA, Altome SA. Prevalence of protozoa and gastrointestinal helminthes in stray cats in Zanjan province, north-west of Iran. Iranian J Parasitol. 2009;4(3):71-5.

28. Sharif M, Nasrolahei M, Ziapour SP, Gholami S, Ziaei H, Daryani A, et al. Toxocara cati infections in stray cats in northern Iran. $J$ Helminthol. 2007;81(1):63-6.

29. Motazedian H, Mehrabani D, Tabatabaee SH, Pakniat A, Tavalali M. Prevalence of helminth ova in soil samples from public places in Shiraz. East Mediterr Health J. 2006;12(5):562-5.

30. Saraei M, Zakilo M, Tavazoei Y, Jahanihashemi H, Shahnazi M. Contamination of soil and grass to Toxocara spp. eggs in public parks of Qazvin, Iran. Asian Pacific J Trop Biomed. 2012;2(2):S1156-8.

31. Tavalla M, Oormazdi H, Akhlaghi L, Razmjou E, Lakeh MM, Shojaee S, et al. Prevalence of parasites in soil samples in Tehran public places. Afr J Biotechn. 2012;11(20):4575-8.

32. Zibaei M, Abdollahpour F, Birjandi M, Firoozeh F. Soil contamination with Toxocara spp. eggs in the public parks from three areas of Khorram Abad, Iran. Nepal Med Coll J. 2010;12(2):63-5.

33. Blaszkowska J, Wojcik A, Kurnatowski P, Szwabe K. Geohelminth egg contamination of children's play areas in the city of Lodz (Poland). Vet Parasitol. 2013;192(1-3):228-33.

34. Bahrami A, Doosti A, Nahravanian H, Noorian AM, Asbchin SA. Epidemiological Survey of Gastro-Intestinal Parasites in Stray Dogs and Cats. Aus J Basic Appl Sci. 2011;5(9).

35. Barutzki D, Schaper R. Results of parasitological examinations of faecal samples from cats and dogs in Germany between 2003 and 2010. Parasitol Res. 2011;109 Suppl 1:S45-60. 
